\title{
BMJ Open Radiologists' perspectives about evidence-based medicine and their clinical practice: a semistructured interview study
}

\author{
Allison Tong, ${ }^{1,2}$ Suzanne E Mahady, ${ }^{3}$ Jonathan C Craig, ${ }^{1,2}$ Gabes Lau, ${ }^{4}$ \\ Anthony J Peduto, ${ }^{5}$ Clement Loy ${ }^{1}$
}

To cite: Tong A, Mahady SE, Craig JC, et al. Radiologists' perspectives about evidencebased medicine and their clinical practice: a semistructured interview study. BMJ Open 2014;4: e006199. doi:10.1136/ bmjopen-2014-006199

- Prepublication history and additional material is available. To view please visit the journal (http://dx.doi.org/ 10.1136/bmjopen-2014006199)

Received 23 July 2014 Revised 14 November 2014 Accepted 17 November 2014

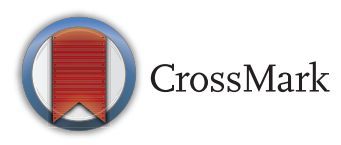

For numbered affiliations see end of article.

Correspondence to Dr Allison Tong; allison. tong@sydney.edu.au

\section{ABSTRACT}

Objectives: To describe radiologist's attitudes and perspectives on evidence-based medicine (EBM) and their practice.

Design: Face-to-face semistructured interviews, thematic analysis.

Setting: 24 institutions across six Australian states and New Zealand. Transcripts were imported into HyperRESEARCH software and thematically analysed.

Participants: 25 radiologists.

Results: Six themes were identified: legitimising decisions (validated justification, prioritising patient preferences, reinforcing protocols), optimising outcomes (ensuring patient safety, maximising efficiency), availability of access (requiring immediacy, inadequacy of evidence, time constraints, proximity of peer networks, grasping information dispersion), overriding pragmatism (perceptibly applicability, preserving the art of medicine, technical demands), limited confidence (conceptual obscurity, reputation-based trust, demands constant practice, suspicion and cynicism), and competing powers (hierarchical conflict, prevailing commercial interests).

Conclusions: Radiologists believe EBM can support clinical decision-making for optimal patient outcomes and service efficiency but feel limited in their capacities to assimilate and apply EBM in practice. Improving access to evidence, providing ongoing education and training supplemented with practical tools for appraising evidence; and developing evidence-based guidelines and protocols may enhance feasibility and promote the confidence and skills among radiologists in applying EBM in radiology practice for better patient care.

\section{INTRODUCTION}

Evidence-based medicine (EBM) is the "integration of best research evidence with clinical expertise and patient values" ${ }^{2}$ and is widely promoted as a tool to improve patient care. EBM was originally designed to address clinical problems within internal medicine, but has been generalised and expanded to

\section{Strengths and limitations of this study}

- Interviews were conducted until saturation and we recruited participants from a range of demographic characteristics, years of practice in radiology, and evidence-based medicine training.

- Preliminary findings were sent to the participants for to ensure that the results reflected the full breadth of their perspectives.

- Participants were recruited from two countries and therefore transferability to other countries beyond Australia and New Zealand is uncertain.

enable applicability to other medical specialties. Recognised barriers to the optimal use of EBM in a variety of specialties include competing priorities and lack of time, inability to cope with 'information explosion', ${ }^{3}$ misconceptions about what constitutes quality evidence, ${ }^{4}$ lack of awareness of EBM resources, ${ }^{3}$ threat to professional autonomy, ${ }^{5}$ concerns about the trustworthiness of the data, ${ }^{5}$ fear of conflict with colleagues, ${ }^{7}$ and institutional dynamics and culture. ${ }^{8-10}$ However, there is little information on how EBM is regarded and practised by radiologists and whether barriers to optimal use may differ.

For example, technological innovation in imaging and interventional technologies has intensified the demand on radiologists to assimilate the proliferation of new information to deliver safe and cost-effective care, ${ }^{11} 12$ and the promotion of new tests may occur prior to published evidence supporting widespread use. EBM use within radiology has not been examined, but a survey of radiation oncologists found widespread support for EBM, although most had not undertaken formal EBM training and were unaware of integral resources such as the Cochrane Library. ${ }^{3}$

We aimed to describe radiologist's attitudes and perspectives on learning and applying 
EBM in practice, and in doing so, inform strategies for improving EBM training, and assist development of resources to facilitate greater use of EBM. This may help to foster appropriate decisions regarding imaging and interventional procedures for improved patient outcomes.

\section{METHODS}

Participant selection and practice setting

Radiologists in Australia and New Zealand were purposively selected to capture a range of age, gender, years of clinical experience, radiology subspecialties, EBM training, and practice locations. Invitations were emailed to radiologists enrolled in the critical skills appraisal programme at The University of Sydney (July 2013) or were attending an Annual Scientific Meeting (October 2013). Radiologists known to the investigators were also invited and participants could nominate other radiologists who could offer a different perspective about EBM. Interviews were conducted in meeting rooms, clinic offices and conference venue. All participants provided informed consent.

\section{Data collection}

The interview guide was based on a review of the literature $^{3-8} \quad 13 \quad 14$ and discussion among the research team (box 1). AT conducted a face-to-face semistructured interview with each participant from July to November 2013. Participant recruitment ceased when theoretical saturation was reached that is, when little or no new concepts were being raised in subsequent interviews. All interviews were audio-recorded and transcribed.

\section{Analysis}

The transcripts were entered into HyperRESEARCH software (ResearchWare, US V.3.0, Randolph Massachusetts, USA) for coding qualitative data. Based on the principles of grounded theory (to develop a theory or explanation ie, grounded in the data collected ${ }^{15}$ and thematic analysis, AT read the transcripts, conceptualised and coded all sections relating to radiologists' perspectives on EBM into concepts identified inductively in the data; and created new codes when necessary. These were reviewed by SEM who also read the transcripts. This can help to ensure that data were captured in the preliminary codes. Similar concepts were grouped into themes and subthemes. Patterns and conceptual links between themes were mapped into a thematic schema. Member checking was conducted whereby participants were sent a copy of the preliminary analysis and given 2 weeks to suggest

\section{Box 1 Interview guide}

\section{Role as a radiologist/radiology trainee}

A. What is your current position and how many years have you been working in that role or what year of training?

B. Working as a radiologist/trainee, what are the some of the most difficult, common, or complex issues/decisions you have faced and how do you deal with them (eg, when interpreting images)?

2. Knowledge about evidence-based medicine (EBM)

A. How would you define EBM or what is the first thing that comes to mind when thinking about EBM?

B. How you rate your knowledge or understanding about EBM from 0 (lowest) to 10 (highest-most knowledgeable)—why?

C. What educational sources do you use to inform your practice? What resources or how do you go about interpreting diagnostic tests? (STATdx, up-to-date, guidelines and protocols, experience and intuition, other colleagues' opinions, decision aids or online resources such as calculators)

D. Can you comment on the level of trust you have in these resources-what makes you trust it more/less? (journal reputation, authors, sample size)

E. What aspect of EBM do you find most challenging or difficult-why? (asking answerable questions, searching, appraising, analysis or synthesis, interpreting the data, applicability to individual patients or applying EBM in your day-to-day practice)

F. What EBM concepts of terms do you feel you understand the least/most?

3. EBM training

A. What was the most/least interesting or valuable thing you learnt in EBM-why?

B. How you rate the importance of EBM training to your-clinical practice from 0 (least) to 10 (most important)-why?

C. How would you improve EBM teaching that is, what teaching methods do you believe would be most effective in helping radiologists apply EBM in their practice-why? (journal club, study appraisal)

4. Applying EBM in practice

A. Can you describe the role EBM has in your overall clinical decision-making? Have you applied EBM in practice-how/give an example?

B. Do you believe there are benefits in applying EBM in radiology practice-why? (service efficiency, costs, patient care, develop guidelines)

C. What are the challenges or barriers in applying EBM in day-to-day practice? (service provision vs consultative, not sure where to find resources to guide its use (access), unsure of the validity of a checklist compared with clinical reasoning, competing priorities, concern about the trustworthiness of the evidence, threat to clinical autonomy, financial interests, potential to cause conflict, contradictory data, information overload)

D. What can you suggest would facilitate the application of EBM into day-to-day practice? (Evidence summaries, technology and tools)

E. Do you think EBM is more relevant for the clinical aspects of radiology such as interventional radiology rather than diagnosis-why?

F. Compared with other medical specialties, do you think radiology lags behind, is equal or, or is more advanced in terms of EBM knowledge and application - why? (research culture, limited research evidence)

G. Does learning about EBM make you more or less likely to undertake research to answer clinical issues you have encountered—why? 
additional opinions. These were integrated into the final thematic analysis. This ensures that the findings reflect and captures the full breadth of data from the participants.

\section{RESULTS}

Twenty-five radiologists from 24 institutions across six Australian states and New Zealand participated. Non-participation $(n=6)$ was due to travel and clinical commitments. The mean duration of interviews was 35 min. Participant characteristics are provided in table 1.

We identified six major themes: legitimising decisions, optimising outcomes, availability of access, over-riding pragmatism, limited confidence, and competing powers. Illustrative quotations for each theme are provided in table 2/online supplementary file 1 . A thematic schema illustrating the conceptual links among themes is shown in figure 1. EBM was believed to support clinical decision-making for optimal patient outcomes and service efficiency, but radiologists' capacities to assimilate and apply EBM were limited by barriers to accessing and appraising the evidence, perceived need for pragmatism and gaining practical experience, and contending with power hierarchies with referring physicians and commercial interests. A description of the themes and subthemes are provided in the following section. Most of the themes apply to interventional and diagnostic radiology; however results that were specific to either interventional (therapeutic) or diagnostic radiology will be indicated.

\section{Legitimising decisions \\ Validated justification}

EBM provided a framework to make clinical decisions based on science, rather than anecdotal data. EBM "added weight, added experience and evidence behind decisions." And some participants felt reassured when research "validated their own experiences", and EBM was regarded as an opportunity "to borrow information and techniques from other people who have been using them more."

\section{Prioritising patient preferences}

Shared decision-making was regarded as important though some felt that patient preferences contradicted EBM. For example, they felt unable to deny patients who wanted treatment even when the evidence suggested that the therapeutic intervention would not be cost-effective or beneficial.

\section{Reinforcing protocols}

EBM was regarded as useful for developing evidencebased protocols and guidelines. This was particularly relevant in directing decisional pathways for inexperienced referrers. Protocols were seen to protect radiologist's decisions as "you get in trouble for missing something, you don't get in trouble for over-investigating something."
Table 1 Participant characteristics

\begin{tabular}{|c|c|c|}
\hline $\begin{array}{l}\text { Participant } \\
\text { characteristics }(n=25)\end{array}$ & $\begin{array}{l}\text { Number of } \\
\text { participants }\end{array}$ & Per cent \\
\hline \multicolumn{3}{|l|}{ Sex } \\
\hline Men & 20 & 80 \\
\hline Women & 5 & 20 \\
\hline \multicolumn{3}{|l|}{ Age (years) } \\
\hline $30-39$ & 3 & 12 \\
\hline $40-49$ & 11 & 44 \\
\hline $50-59$ & 5 & 20 \\
\hline $60-69$ & 6 & 24 \\
\hline \multicolumn{3}{|l|}{ Years of practice in radiology } \\
\hline$\leq 10$ & 5 & 20 \\
\hline $11-20$ & 10 & 40 \\
\hline $21-30$ & 6 & 24 \\
\hline$>30$ & 4 & 16 \\
\hline \multicolumn{3}{|c|}{ Training in evidence-based medicine } \\
\hline Yes $^{*}$ & 12 & 48 \\
\hline No & 13 & 52 \\
\hline \multicolumn{3}{|l|}{ Radiology subspecialty† } \\
\hline Breast imaging & 3 & 12 \\
\hline Cardiovascular & 8 & 32 \\
\hline Chest & 7 & 28 \\
\hline Emergency & 9 & 36 \\
\hline Gastrointestinal & 8 & 32 \\
\hline General radiology $\ddagger$ & 4 & 16 \\
\hline Genitourinary & 8 & 32 \\
\hline Head and Neck & 6 & 24 \\
\hline Interventional & 11 & 44 \\
\hline Musculoskeletal & 13 & 52 \\
\hline Neuroradiology & 9 & 36 \\
\hline Nuclear radiology & 3 & 12 \\
\hline Obstetrics and gynecology & 1 & 4 \\
\hline Paediatric radiology & 5 & 20 \\
\hline Vascular & 1 & 4 \\
\hline \multicolumn{3}{|l|}{ Type of practice } \\
\hline Public & 23 & 92 \\
\hline Private & 2 & 8 \\
\hline
\end{tabular}

Optimising outcomes

Ensuring patient safety

Some were convinced that EBM had demonstrable impact on ensuring safe patient care. There was reference to studies assessing safe dosages of gadolinium in patients with renal impairment, reducing the incidence of nephrogenic systemic fibrosis. Participants remarked on the "increasing number of normal examinations which meant they were scanning more people, and that they were not selecting them correctly." They felt a burden of responsibility-"we definitely do cause the public harm, we just don't know how many [malignancies] is due to us yet" and believed that applying EBM in diagnostic radiology could reduce overexposing patients to unnecessary ionising radiation and risk of malignancies. 
Table 2 Illustrative quotations

Theme

Validated justification

Prioritising patient preferences

Reinforcing protocols

Optimising outcomes

Ensuring patient safety

Maximising efficiency

Availability of access Requiring immediacy

Inadequacy of evidence

Time constraints

Proximity of peer networks

\section{Illustrative quotations*}

I suppose the antithesis would be anecdotal medicine, so you're getting away from what you perceive, or what you think is the correct way to behave, what you had thought before. What you're trying to do is be critical about what you do and see if there's scientific basis to support the way you practise medicine. (ID08)

The most valuable is that if you can confront a clinician and say that a certain practice is the right practice. To do a certain test in a certain scenario, if you have evidence that shows that that's the best practice then it's easier to stand your ground and then say we shouldn't be doing this and we should be doing it the other way because there is research that suggests that's the best practice. If you don't have that sort of research it's very hard to back up your opinion. (ID05) So it's nice when research validates your own experiences. (ID17)

How can I deny a patient treatment? That's the problem with it. Even if the evidence-based medicine says you shouldn't treat this patient. For example you are a patient, I can't say, this evidence says you shouldn't be treated but the patient still wants it, what do you do? That's the ethical dilemma. (ID15)

We have in the department guidelines to help people to request certain radiology procedures on the basis of what was published. This is the evidence that you should go down this pathway and don't ask for anything more. (ID21)

We would decrease the amount of ionisation radiation as we give to the public, which hopefully then would decrease the number of malignancies that we cause. We definitely do cause them, given on a population level, we just don't know how many is due to us, yet. (ID04) Perhaps one area where evidence-based medicine has had a benefit in paediatrics is the reduction in imaging for vesicoureteral reflux. There's been no improvement or change in the incidence of chronic renal disease in children as a consequence of treatment of urinary tract infection or treatment of reflux. So that has totally changed the management. Seven or eight years ago I was doing MCUs on children up to five years old and there were probably five or 10 on a list. Now it's down to less than one because of evidence-based medicine showing that the treatment doesn't-the investigation and treatment doesn't change outcome. (ID13)

So the literature that appeared over that period of time has really changed the way that I think about using gadolinium in renal impairment. I'm not as scared to use it anymore providing I stick to guidelines with regard to the estimated GFR. (ID25)

Service efficiency, there is a safety aspect in that they don't have to undergo other tests as well, more invasive tests, and economic from the point of view that they're not taking up further resources in the hospital which could be used for other people. (ID01)

If I really need an article that is not available I get the library to get it for me, but that adds an extra element of difficulty, so I tend to just ignore the ones-not ignore, but I tend to find a free access version if I can, not of the same study but as best I can. (ID04)

MEDLINE- unfortunately a lot of the time you can't get in journals what you want and a lot of the time they only give you the abstracts there, so that's where the Google comes into it, because HighWire provides a lot of free journals there. You can actually go to the full journal rather than just the abstract. I guess I can always go to the university library and find out but it takes a long while to get in there. (ID24)

The technology's there and you're playing catch up really. It jumps ahead, the lag is apparent. (ID07) Often the outcomes aren't measurable. So you may see certain findings but you'll never find out because that patient doesn't have the joint opened up or-so the outcome that you're measuring or the gold standard, there may not be a gold standard in a lot of the studies-where you have surgical proof or autopsy proof. (ID25)

It's a lot of time so sometimes it's obviously easier just to say it needs follow-up or repeat imaging rather than taking time out to consult a study. (ID13)

I guess you can't be really a true academic because the clinical service is so demanding. You just don't get time to do it. (ID24)

Colleagues' opinions, because l'm doing some interventional stuff, and often, the interventional has much less research. If you get into trouble, try this, and, have you thought about doing this, and, here's another approach. They are not the stuff that gets written up. It's more just because it's a "doing" rather than a "thinking about" bit. So, for those things, talking to colleagues is much more important. (ID11) 
Table 2 Continued

Theme Illustrative quotations*

I haven't had any formal training in EBM. I don't actively practise EBM, I don't visit the Cochrane Institute. I'm aware of it but I don't actually regularly view it to see what's out there. My approach to medicine is a very practical approach and based on my experience and the knowledge of others. My skill set is complementary to others, so I use their skills. I'm not the sort of person who remembers detailed differential diagnoses or percentages but I know that that's not a good finding or a good finding or it requires this person to review and share their knowledge. We each have different skill sets. That's how I see my role. (ID13)

Grasping information dispersion

Over-riding pragmatism Perceptible applicability

Preserving the art of medicine

Technical demands

Limited confidence Conceptual obscurity

Reputation-based trust

Demands constant practice

Suspicion and cynicism
Because radiologists in most places are generalists, so you have to be able to do neuro, you have to be able to do gastro, you have to be able to do intervention, it's not actually possible to be at the top level of science in all those fields. (ID07)

It's very hard to practice in a non-Google fashion in all of those fields. In some areas you'll be able to or if that's all you do you can, because you'd be up with the literature and you'll know about it otherwise you're kind of just going with a level of safety that's acceptable, but it's probably not the top end of care. (ID07)

[EBM] is completely foreign to my brain and I'm afraid that's why I haven't bothered to learn how to evaluate these things in a statistical analysis way...it's certainly not something I use for my day to day work. (ID14)

I don't read journal articles to know about evidence based medicine per se, like meta-analyses find less instantly useful. You have to spend a lot more energy on trying to pick out a tiny little fragment of useful data, so most of the time the article was justifying itself and talking about itself. I just sort of get to the crux of the matter, so yeah. (ID14)

I look at their protocol in terms of what they do and then whether their outcome measures more than probably analysing the way they got the outcomes. (ID07)

I'm not a boffin, I'm not an academic, I'm much more practical, and I'm not saying academics aren't practical.... I'm more operational. (ID23)

They are probably more like spending time reading about things-learning from practice is more important than reading from it. You see a patient and if you make a mistake and know that you made a mistake you'll never do that again. But that is real medicine. That's real evidence-based medicine I tell you. (ID24)

If everything gets based on evidence-based medicine we lost the art-this is my one piece of information you is that we lost the art of radiology...I don't think that is should be rigid. I think there needs to be room for the art of medicine. (ID14)

It's not looking for research articles, it's looking for information. As I said what does such and such a condition look like on ultrasound? You don't need a research article. (ID12)

So I guess it's becoming more and more complex rather than just a film that you can read, but you have to go and find out how to do it and get the right sequences done and then on top of it you have to relearn your anatomy, because normally you don't see cartilage and soft tissues on other modality but the MR are coming on where you can see everything, so you have to know the tiny gritty bits there and capsules, the tendons and normally in old times you don't see it. So it's a fast-growing field that you have to keep on learning new tricks there, as well as refine what you knew before. (ID24)

I think we all aspire to practice it but we don't necessarily know how to and I think there's a lot of quasi-I'm not a victim and a perpetrator of it but there's a lot of quasi EBM going on. (ID07) if you asked me to list the different types of bias, I would have had great difficulty doing it. Just because l've never actually sat and thought through a framework. (ID03)

If it comes from a reputable journal within radiology...I would give more validity to or I tend to say oh well obviously if it's got through the editors it must be good. (ID08)

Certainly, going through critical assessment of particular papers, I think those sorts of exercises do develop our understanding and confidence in appraisal with more experience. I guess even in the department, having journal clubs and again, practising critical appraisal would help everyone in our department. (ID01)

Lies, more lies, and statistics. You can make anything into anything when you know statistics. (ID 04) I've seen a few cases now where different meta-analyses will draw completely different conclusions from the same set of data analysing the same group of papers. (ID20) 
Table 2 Continued

\begin{tabular}{ll}
\hline Theme & Illustrative quotations* \\
\hline In my personal experience people usually decide what they want the reality to be and then \\
harness the appropriate evidence that they want to support it, particularly in imaging. (ID20) \\
$\begin{array}{l}\text { Competing powers } \\
\text { Hierarchical conflict }\end{array}$ \\
So we not control the ultimate management of the patient because we are secondary referrals, \\
I mean there's a bit of a stigma in radiology, it's seen by some other specialties as being a bit \\
of a service profession or service industry to the rest of medicine, in that we're just doing tests \\
because the others decide that that test needs to be done that we do. (ID12) \\
The doctors are covering their backside and being defensive; they're also being efficient. Right, \\
you know it's quicker to get us to do a scan than it is to wait for the surgical registrar to get out \\
of theatre and come and see the patient. And from their point of view it's probably also being of \\
the patient's advocate in saying, I don't care if this only benefits one patient in a hundred; you \\
do it for my patient. I'm just letting you know EBM to radiologists, it has that theme to it like it's \\
all wonderful. But it's impossible for us to actually use. I shouldn't say impossible because \\
that's an exaggeration, but it's difficult. (ID02) \\
Radiology, cardiology, endoscopy, all the various other things where somebody gets paid for \\
doing something, the temptation is to go and do it. You can always justify it to a certain extent. \\
(ID18)
\end{tabular}

\section{Maximising efficiency}

Participants felt certain that the financial costs to public hospitals incurred by performing excessive radiological tests and procedures could be minimised by applying EBM. Some suggested that evidence-based referral guidelines may reduce the number of unnecessary diagnostic tests ordered by referrers and improve economic and service efficiency.

\section{Availability of access}

Requiring immediacy

Simple and direct access to information was important. Most used Google or PubMed as their primary search engine. Some relied on email updates or review articles to keep up-to-date. Evidence summaries offered information that "had already been critically appraised, filtered out so most of the rubbish wasn't there." Being unable to access journals due to institutional restrictions "added an extra element of difficulty" however, some participants who had received EBM training felt confident about searching for high-quality scientific research using MEDLINE and the Cochrane Library.

\section{Inadequacy of evidence}

Radiological technologies often "progressed before the evidence could come out." Some observed that diagnostic procedures (eg, CT angiograms for vertebral artery dissection) were used without supporting evidence. In radiological research, some felt that relevant outcomes could not be feasibly measured, for example if it necessitated surgical or autopsy proof. Research in diagnostic imaging was unavailable particularly for rare clinical cases. They felt that, "there's no culture of [radiology] research except in little pockets and enclaves in different institutions few and far between."

\section{Time constraints}

The 'fast-growing field' of radiology meant participants felt without capacity to be "a true academic," engage in research, and "to go through everything we do and find the evidence to prove that that's the best method or way of doing something." Instead of an appointment-based patient caseload, radiologists had to manage a continuous inflow of patients, which was more difficult to keep manageable particularly if working in a smaller radiology department, or in the private sector. Some reviewed the literature only when required to, for example when preparing for presentations.

\section{Proximity of peer networks}

Participants relied on their colleagues to discuss and resolve cases. In larger radiology departments, participants could readily contact experienced colleagues for advice. One participant stated, "My approach to medicine is a very practical approach and based on my experience and the knowledge of others, my skill set is complementary to others so I use their skills." Interventional radiologists valued practical suggestions from colleagues about procedures and participants attended conferences to learn about new procedures and protocols, then turned to "selected articles on the nuts and bolts, assuming that somebody has looked at the utility of the procedure."

\section{Grasping information dispersion}

The field of radiology was described as broad, "dynamic," and all-encompassing of different subspecialties which augmented the challenge of keeping up with the literature-"radiologists in most places are generalists, you have to be able to do neurology, gastroenterology, intervention, it's not actually possible to be at the 
Perceived benefits of evidence-based medicine

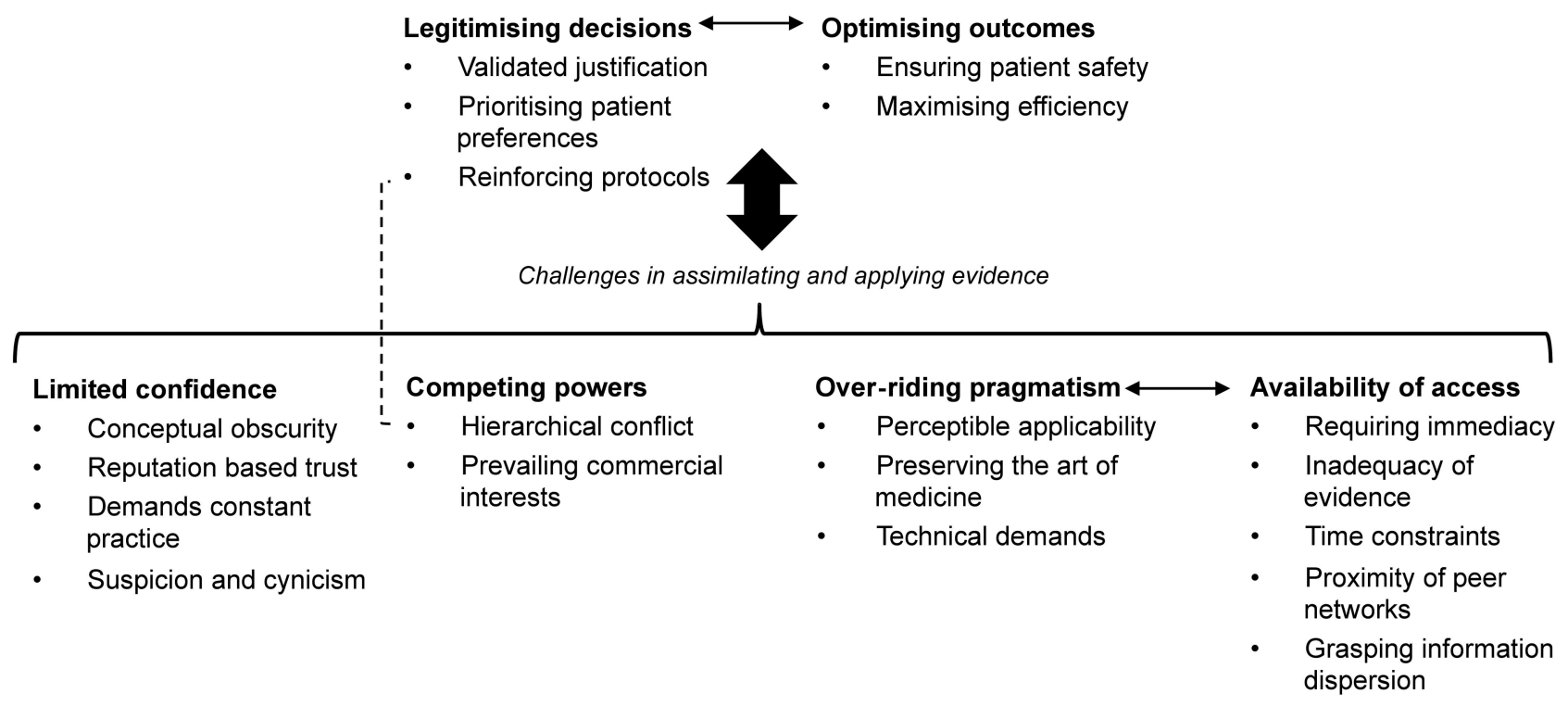

Figure 1 Thematic schema.

top level of science in all those fields." They felt "confounded by the plethora of information that you can't filter anymore." Radiologists practising within a narrow subspecialty felt better able to remain aware of the current literature in their area.

\section{Over-riding pragmatism}

Perceptible applicability

From a practical perspective, some judged the clinical relevance of a research article rather than the methodology. One participant reflected, "I'm not an academic, I'm much more practical, I'm more operational." They would "look at what they do and the outcomes measures more than analysing the way they get the outcomes." Research results that were too broad or excluded relevant patient groups made it difficult to extrapolate or assess the transferability of the findings to their own patient population or to an individual patient-"like meta-analysis, you have to spend more energy on trying to pick out tiny fragments of useful data, so most of the time the article was justifying itself and talking about itself, I just want to get to the crux of the matter."

\section{Preserving the art of medicine}

There was anxiety that "if everything gets based on evidence based medicine, we lose the art of radiology." Participants with more years of experience believed in learning from practice, observing senior colleagues, and developing expert intuition, more so than 'reading about things' as EBM could 'never capture the whole story.'

\section{Technical demands}

The technological advances in radiology placed demands on participants to prioritise their technical competence and knowledge of anatomy and pathology.
They had to "keep on learning new tricks, as well as refine what they knew before" and study textbooks and 'didactic' articles rather than scientific research publications-"What does such a condition look like on ultrasound? You don't need a research article."

\section{Limited confidence}

\section{Conceptual obscurity}

The perceived complexity of concepts, mainly relating to critical appraisal and statistical analysis, was overwhelming. Many described their judgement about study validity as 'superficial' and felt they lacked a framework for critically appraising an article- "we all aspire to practice EBM but we don't necessarily know how to and I think there's a lot of quasi EBM going on." Some assessed articles based on sample size, participant characteristics, and blinding. Also, some believed a high-level understanding of statistics was required for EBM, perceived to be daunting, "we've come out of it with this monster that most of the radiologists don't know how to cope with, most of us left statistics behind in high school, we don't have this analysis in our brains."

\section{Reputation-based trust}

Participants trusted journals with high impact factors, and articles from reputable institutions with experienced authors. They placed confidence in editorial integrity and expertise to ensure that only high-quality and valid research articles were published.

\section{Demands constant practice}

EBM was a skill that required ongoing practice. Participants suggested regular EBM training, and journal clubs to maintain EBM proficiencies, such as conducting critical appraisal. 


\section{Suspicion and cynicism}

Some were suspicious of authors who might be misusing research to push their own agenda, "People usually decide what they want the reality to be and then harness the appropriate evidence that they want to support it." For example, in diagnostic cardiac imaging, one participant observed nuclear physicians advocating for nuclear medicine while cardiologists were promoting stress echocardiogram, and both presented compelling arguments supported by research. Contradictory results also perpetuated cynicism of EBM, "I've seen a few cases where different meta-analyses will draw completely different conclusions from the same set of data analysing the same papers." Some were wary of academic competition, and the "politics of journals, and personal egos.' However, multiple independent studies which demonstrated confirmatory findings, provided reassurance.

\section{Competing powers}

Hierarchical conflict

Disempowerment prevented the practice of EBM. Some radiologists felt that referring physicians perceived them as service providers rather than as consultants. They viewed that "referrers don't feel that radiology should be gate keepers." Being 'secondary referrals' most radiologists did not control patient management and lacked clinical information about the patient. Some resigned themselves to 'defeat' and 'dogmatism' as they continued to perform tests they regarded as unnecessary. At times, there was a palpable tension between keeping referrers satisfied and advocating for the patient's safety and preventing them from being "irradiated just for expediency rather than a clinical indication". Some tried to 'battle' with referrers but withdrew from the "uneven playing field". More senior participants felt that referrers respected their recommendations.

In certain radiology subspecialties including paediatrics, oncology, and obstetrics, radiologists participated in multidisciplinary meetings and valued the active engagement in patient management where their expert opinion contributed to the broader decision-making. Participants appreciated this 'cross-pollination' of information and clinical history.

\section{Prevailing commercial interests}

Private radiology centres faced the pressure of "generating revenue to keep the practice going", which was perceived to nullify any impetus to implement EBM. Some believed that "evidence-based medicine will never work in an item for service based medical culture" and there was "no real incentives for doctors to do the right thing" in referring patients for radiological diagnostics tests. To protect a thriving business, they kept referrers satisfied by fulfilling their radiological requests, even when it was not evidence based.

\section{DISCUSSION}

Although radiologists appreciate the role of EBM in improving patient care, misperceptions of the definition of EBM, a lack of critical appraisal skills and an underappreciation of how EBM could help resolve common tensions within daily practice limited its optimal use. EBM is defined as the integration of best research evidence with clinical expertise and patient values however some participants thought that EBM supplanted clinical expertise and therefore rejected it as being exclusive of clinical wisdom. A common tension cited by many participants was the performance of unnecessary tests, contributing to excess cost and increased exposure to radiation, however many felt helpless to refuse the request. However, when evidence-based guidelines were available to support appropriate imaging pathways, radiologists felt more confident in negotiating referrals.

Some of the barriers to implementing EBM we identified have been reported in other areas of medicine and health. Studies conducted in internal medicine and surgery found that confusion about EBM terminology, team dynamics, staff disapproval, and time constraints prevented residents from practising EBM. ${ }^{7}{ }^{8} \mathrm{In}$ primary care, EBM was perceived by some physicians as devaluing the 'art' of medicine and a threat to their professional autonomy, and were concerned about industry influence. ${ }^{5}$ Another study found that healthcare providers preferred tested, convenient and respected evidence sources including professional societies and expert colleagues. ${ }^{4}$ There are unique features in radiological practice: the limitations of being perceived as a service provider rather than an 'expert consultant', the demands of maintaining technical competence, the requirement for detailed but stable knowledge of anatomy and pathology, and the challenges of keeping up in a field of rapid technological advances.

While understanding and use of EBM is widely accepted as a core competency of clinical practice, this is the first study to explore understanding and barriers to use in radiology. We conducted interviews until little or no new concepts were emerging from subsequent interviews (theoretical saturation), and included participants from a range of demographic characteristics, years of practice in radiology, and EBM training. Also, participants were asked to provide feedback on the preliminary findings (member checking). However, our study has potential limitations. Participants were recruited from Australia and New Zealand therefore the transferability of the findings to other regions may be limited, although similar barriers have been identified in studies conducted in different settings, ${ }^{16}$ suggesting broader applicability.

The acquisition and application of EBM skills including literature searching, critical appraisal of articles and interpretation of diagnostic tests and their limitations is essential to competent clinical care. ${ }^{12}$ Several resources have been published in radiology literature. ${ }^{12}{ }^{17}$ 
Table 3 Suggested strategies for promoting EBM in radiology

\begin{tabular}{|c|c|}
\hline Key areas of improvement & Suggested strategies and action \\
\hline $\begin{array}{l}\text { Quality and quantity } \\
\text { of evidence }\end{array}$ & $\begin{array}{l}\text { Fostering a research culture (eg, clinician researcher interaction) } \\
\text { Link to academic institutions } \\
\text { Form multicentre research networks } \\
\text { - Focus on the evidence base should be an integral part of multidisciplinary meetings }\end{array}$ \\
\hline Access to evidence & - Mobile applications \\
\hline Education and training & $\begin{array}{l}\text { Definition of EBM, emphasising the role of clinical expertise and patient preferences in EBM } \\
\text { Explain the pros and cons of Google and PubMed searches or other sources (STATdx) } \\
\text { Clarify the fundamentals of EBM being the evidence hierarchy } \\
\text { Demonstrate practice tips for using Cochrane to answer a clinical question } \\
\text { Provide a framework for critical appraisal } \\
\text { Setting EBM in context (not sacrificing clinical experience or the art of medicine) } \\
\text { Mandated training for example, CME } \\
\text { Short courses in EBM (+ online support) }\end{array}$ \\
\hline Applying EBM in practice & $\begin{array}{l}\text { Journal club meetings (include tools, feedback)-face-to-face or online } \\
\text { Ongoing training in using EBM, with assistance from experts such as clinical } \\
\text { epidemiologists } \\
\text { Develop and disseminate evidence-based guidelines and protocols for referrers } \\
\text { - Develop evidence summaries in clinical practice } \\
\text { Promote access to preappraised evidence-based resources such as AJR guidelines }\end{array}$ \\
\hline Empowerment & Conduct audits of referral practices \\
\hline
\end{tabular}

However, barriers related to the availability and access to evidence, unmet education and training needs, pragmatic and structural difficulties that need to be addressed. Based on our findings, we suggest key target areas, strategies and actions for promoting EBM awareness and implementation (table 3 ).

Moving EBM teaching from the classroom to clinical practice settings has been strongly advocated to improve knowledge, critical appraisal skills, attitudes and behaviour. ${ }^{18}$ The few strategies to clinically integrate EBM teaching which have been evaluated include daily EBM teaching rounds in which searches and study appraisals are based on cases presented at clinical rounds, ${ }^{19}$ journal clubs, ${ }^{20}$ and EBM ward round sessions led by a clinical specialist and epidemiologist to develop clinical questions, literature search, critical appraisal and development of evidence summaries. ${ }^{21}$ While these teaching methods are effective, more specific evaluation of these strategies is needed in radiology.

It has been recognised that evidence-based practice should be taught from an early stage in medical and radiological education. $^{22} 23$ Current models for educating evidence-based practice include having trained epidemiologists to deliver regular teaching and interactive sessions which encompass theory, self-directed learning, and application to clinically relevant questions; or providing training workshops or teaching EBM in postgraduate meetings which cover the principles of EBM. ${ }^{22}$ Our study has highlighted important implications for teaching EBM within radiology. Skills that require greater emphasis included literature searching and critical appraisal. A recent survey found that radiologists and trainees preferred Google, customised radiologyfocused produces and apps, and online resources to solve imaging questions. ${ }^{16}$ Evidence summaries or EBM guidelines could be developed and disseminated via these channels. Confidence in appraisal skills could be improved with wider use of EBM tools. ${ }^{24}$ For most EBM skills, a mix of educational strategies is likely to be most effective in increasing skills including interactive online courses, journal clubs and seminars. ${ }^{25}$

While our findings are likely to have some commonality across geographic regions, further studies on barriers to EBM in different areas would be enlightening. Teaching strategies that are most helpful to radiologists should be clarified, as these may not be the same as those for bedside practitioners. Studies of implementation of evidence-based guidelines for imaging pathways and whether these improve patient's important outcomes and cost are also needed.

Better access to evidence, ongoing education and training supplemented with practical tools for appraising evidence; and developing evidence-based guidelines and protocols may promote optimal use of EBM within radiology, and ultimately translate to better patient care.

Author affiliations

${ }^{1}$ Sydney School of Public Health, The University of Sydney, Sydney, New South Wales, Australia

${ }^{2}$ Centre for Kidney Research, The Children's Hospital at Westmead, Sydney, New South Wales, Australia

${ }^{3}$ Storr Liver Unit, Westmead Millennium Institute, The University of Sydney, Sydney, New South Wales, Australia

${ }^{4}$ Otago Radiology Limited, Pacific Radiology Group, Dunedin, New Zealand ${ }^{5}$ Department of Radiology, Westmead Hospital, Westmead, Sydney, New South Wales, Australia

Acknowledgements The authors sincerely thank all the participants for their time and efforts in sharing their perspectives. With written consent, they 
acknowledge: Roger Bain, Lourens Bester, Roger Bodley, Timothy Cain, Kwang Chin, Craig Hacking, Robin Harle, Albert Lam, Lance Lawler, Melissa Lea, Wai-Kit Lee, Philip Lew, Arthur McKenna, Sabaratnum Muthukumaraswamy, Anthony Peduto, Alex Rhodes, Umesh Shetty, Rohan van den Driesen, Pamela Walsh.

Contributors AT participated in the design of the study, did the interviews, transcribed the interviews, carried out the thematic analysis and drafted the manuscript. SEM, JCC and CL designed the study, participated in the thematic analysis, and provided critical review of manuscript drafts. GL and AJP participated in the design of the study, assisted with the analysis, and provided intellectual input into subsequent manuscript drafts. All authors made substantial contributions to conception and design, acquisition of data, or analysis and interpretation of data; drafting the article or revising it critically for important intellectual content; and provided final approval of the version to be published. All authors had full access to all of the data in the study and can take responsibility for the integrity of the data and the accuracy of the data analysis.

Funding This research is funded by an internal grant from the Sydney School of Public Health, The University of Sydney.

Competing interests None.

Ethics approval The study was approved by the University of Sydney Ethics Committee (20130616).

Provenance and peer review Not commissioned; externally peer reviewed.

Data sharing statement No additional data are available.

Open Access This is an Open Access article distributed in accordance with the Creative Commons Attribution Non Commercial (CC BY-NC 4.0) license, which permits others to distribute, remix, adapt, build upon this work noncommercially, and license their derivative works on different terms, provided the original work is properly cited and the use is non-commercial. See: http:// creativecommons.org/licenses/by-nc/4.0/

\section{REFERENCES}

1. Sackett DL, Resenberg WM, Gray JA, et al. Evidence-based medicine: what it is and what it isn't. BMJ 1996;312:71-2.

2. Sackett DL, Straus SE, Richardson WS, et al. Evidence-based medicine. How to practice and teach EBM. London UK: Harcour Publishers Limited, 2000.

3. Veness M, Rikard-Bell G, Ward J. Views of Australian and New Zealand radiation oncologists and registrars about evidence-based medicine and their access to internet-based sources of evidence. Australas Radiol 2003;47:409-15.

4. Lorenz KA, Ryan GW, Morton SC, et al. A qualitative examination of primary care providers' and physician managers' uses and views of research evidence. Int J Qual Health Care 2005;17:409-14.

5. Tracy CS, Dantas GC, Upshur REG. Evidence-based medicine in primary care: qualitative study of family physicians. BMC Fam Prac 2003;4:1-9.
6. Haynes RB, Devereaux PJ, Guyatt GH. Physicians' and patients' choices in evidence based practice. BMJ 2002;324: 1350.

7. Bhandari M, Montori V, Devereaux PJ, et al. Challenges to the practice of evidence-based medicine during residents' surgical training: a qualitative study using grounded theory. Acad Med 2003;78:1183-90.

8. Green ML, Ruff R. Why do residents fail to answer their clinical questions? A qualitative study of barriers to practicing evidence-based medicine. Acad Med 2005;80:176-82.

9. Richardson WS. We should overcome the barriers to evidence-based clinical diagnosis! J Clin Epidemiol 2007;60:217-27.

10. Kitto S, Petrovic A, Gruen RL, et al. Evidence-based medicine training and implementation in surgery: the role of surgical cultures. J Eval Clin Pract 2011;17:819-26.

11. Evidence-Based Radiology Working Group. Evidence-based radiology: a new approach to the practice of radiology. Radiology 2001;220:566-75

12. Kelly AM, Cronin P. How to perform a critically appraised topic: part 1, ask, search, and apply. AJR Am J Roentgenol 2011:197:1039-47.

13. Jette DU, Bacon K, Batty C, et al. Evidence-based practice: beliefs, attitudes, knowledge and behaviors of physical therapists. Phys Ther 2003;83:786-805.

14. Young JM, Ward JE. Evidence-based medicine in general practice: beliefs and barriers among Australian GPs. J Eval Clin Pract 2001;7:201-10.

15. Strauss A, Corbin J. Basics of qualitative research. Techniques and procedures for developing grounded theory. Thousand Oaks, California: Sage Publications, 1998.

16. Niederhauser BD, Liaw K, McDonald RJ, et al. Pick up a book or "google it?" a survey of radiologist and trainee-preferred references and resources. J Digit Imaging 2014;27:26-32.

17. Budovec JJ, Kahn CE Jr. Evidence-based radiology: a primer in reading scientific articles. AJR Am J Roentgenol 2010;195:W1-4.

18. Coomarasamy A, Khan KS. What is the evidence that postgraduate teaching in evidence based medicine changes anything? A systematic review. BMJ 2004;329:1-5.

19. McGinn T, Seltz M, Korenstein D. A method for real-time, evidence-based general medical attending rounds. Acad Med 2002;77:1150-2.

20. Khan KS, Pakkal M, Brace V, et al. Postgraduate journal club as a means of promoting evidence-based obstetrics and gynaecology. $J$ Obstet Gynaecol 1999;19:231-34.

21. Haines SJ, Nicholas JS. Teaching evidence-based medicine to surgical subspecialty residents. J Am Coll Surg 2003;197:285-9.

22. van Beek EJR, Malone DE. Evidence-based practice in radiology education: why and how should we teach it? Radiology 2007;243:633-40

23. Del Mar C, Glasziou P, Mayer D. Teaching evidence based medicine. BMJ 2004;329:989-90.

24. Guyatt GH, Haynes RB, Jaeschke RZ, et al. Users' guides to the medical literature: XXV. Evidence-based medicine: principles for applying the Users' guides to patient care. Evidence-based medicine working group. JAMA 2000;284:1290-6.

25. Young JM, Rohwer A, Colmink J, et al. What are the effects of teaching evidence-based health care (EBHC)? Overview of systematic reviews. PLoS One 2014;9:e86706. 